\title{
Origin of folded bands in metamaterial crystals
}

\author{
Peter Markoš and Richard Hlubina \\ Department of Experimental Physics, Comenius University, Mlynská Dolina F2, 842 48 Bratislava, Slovakia
}

\begin{abstract}
Recently it has been found numerically that the spectra of metamaterial crystals may contain pairs of bands which disappear inside the Brillouin zone. We observe that the wave equations for such systems are essentially non-Hermitian, but $\mathcal{P} \mathcal{T}$-symmetric. We show that the real-frequency spectra correspond to $\mathcal{P} \mathcal{T}$-symmetric solutions of the wave quation. At those momenta in the Brillouin zone where apparently no solutions exist, there appear pairs of complex-frequency solutions with spontaneously broken $\mathcal{P} \mathcal{T}$ symmetry.
\end{abstract}

PACS numbers: 02.60.Lj, 11.30.Er, 42.70.Qs, 78.67.Pt

One of the basic characteristics of waves propagating in material media is their frequency spectrum. In periodic systems, for instance in photonic crystals, the frequency $\omega$ is a function of the wave vector $\vec{q}, \omega=\omega_{s}(\vec{q})$, where $\vec{q}$ is restricted to an elementary tile of the reciprocal space, the so-called first Brillouin zone. The discrete index $s$ numerates distinct branches of the dispersion, which correspond to different distributions of the wave field within the unit cell of the periodic system. Since in macroscopic systems the wave vector $\vec{q}$ changes quasicontinuously, each branch $s$ leads in general to a finite interval of allowed frequencies, the so-called bands, which may be divided by band gaps in between them [1]

In most systems studied so far, either in the solid-state or photonic context, for each of the branches the function $\omega=\omega_{s}(\vec{q})$ stretches throughout the whole Brillouin zone. This is a simple consequence of Hermiticity. In fact, the plane wave $e^{i \vec{q} \cdot \vec{x}}$ may experience a Bragg scattering to any of the plane waves of the form $e^{i(\vec{q}+\vec{K}) \cdot \vec{x}}$, where $\vec{K}$ is a reciprocal lattice vector. In the basis of such states, the Schrödinger or wave equation takes the form of an eigenvalue problem $H_{\vec{K} \vec{K}^{\prime}}(\vec{q}) c_{\vec{K}^{\prime}}=\lambda(\vec{q}) c_{\vec{K}}$. For a fixed cut-off we are then dealing with an $N \times N$ matrix which, if it is Hermitian, is guaranteed to have $N$ real eigenvalues, independently of the value of $\vec{q}$. Smooth changes of $H_{\vec{K} \vec{K}^{\prime}}(\vec{q})$ lead then to smooth changes of $\lambda(\vec{q})$, resulting in bands which cannot disappear inside the Brillouin zone. In other words, the number of eigenfrequencies cannot be reduced in a certain interval of wave vectors.

However, in numerical simulations it has recently been found that in certain systems the bands may disappear inside the Brillouin zone, forming the so-called folded bands [2]. In particular, such behavior has been observed in photonic crystals in the form of a square array of metamaterial cylinders immersed in vacuum. As an example, in Fig. 1 we show the two lowest-frequency bands for such a metamaterial photonic crystal calculated numerically from transmission spectra [3]. Folded bands appear when the radius of cylinders $R$ increases above the critical value $R_{c} \approx 0.275 a$, where $a$ is the spatial period of the crystal. This surprising result indicates that the wave equation for electromagnetic field in a metamaterial photonic crystal is non-Hermitian 4 .

Actually, it is well known that if both, permittivity $\varepsilon$ and permeability $\mu$, are non-constant functions of the spatial coordinate, then the wave equation for the magnetic field $\vec{H}$ reads

$$
\mathcal{M} \vec{H} \equiv \mu^{-1} \operatorname{rot}\left[\varepsilon^{-1} \operatorname{rot} \vec{H}\right]=\omega^{2} \vec{H},
$$

where the operator $\mathcal{M}$ is non-Hermitian even in ordinary photonic crystals made from dissipationless components [1, 5]. Note that we set the speed of light $c=1$.

So how can it be that folded bands have not been observed in ordinary photonic crystals? The reason is that the non-Hermitian character of Eq. (1) is often not essential, since it can be avoided by a reformulation of the problem. For instance, if the permeability $\mu$ is real and positive definite, one can redefine the magnetic field by $\vec{H}=\vec{h} / \sqrt{\mu}$, thereby transforming Eq. $(1)$ to the form

$$
\mathcal{O} \vec{h} \equiv \mu^{-1 / 2} \operatorname{rot}\left[\varepsilon^{-1} \operatorname{rot}\left(\mu^{-1 / 2} \vec{h}\right)\right]=\omega^{2} \vec{h}
$$

with an explicitly Hermitian operator $\mathcal{O}[1]$.

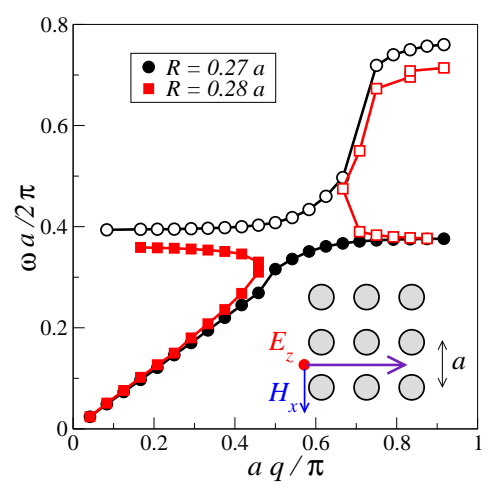

FIG. 1: (Color online) Dispersion relation $\omega=\omega(\vec{q})$ in the $\Gamma X$ direction for a two-dimensional photonic crystal made of metamaterial cylinders with real and dispersionless permittivity $\varepsilon=-1.8$ and permeability $\mu=-5$, see inset. Electric field is taken to be parallel to the cylinders. The two sets of curves correspond to cylinder radii slightly above and below the critical radius $R_{c} \approx 0.275 a[3]$. 


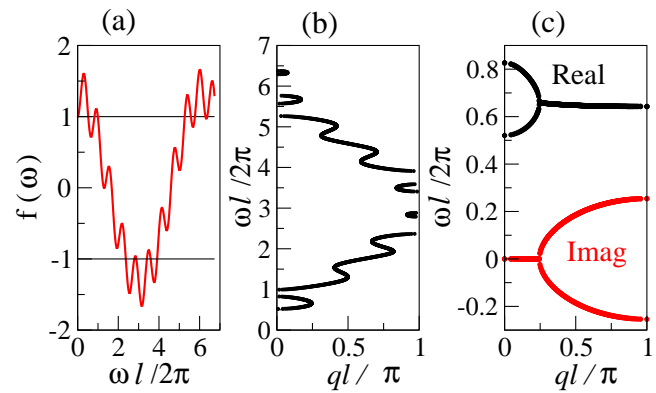

FIG. 2: (Color online) 1D model with $\varepsilon_{a}=1, \mu_{a}=1, \ell_{a}=$ 1 , and $\varepsilon_{b}=-9, \mu_{b}=-1, \ell_{b}=0.41$. (a) Function $f(\omega)$. (b) Frequency spectrum. (c) Real and imaginary parts of frequency in the lowest folded band.

The observation of folded bands in metamaterial photonic crystals therefore suggests that their non-Hermitian character should be essential, i.e. not avoidable by any reformulation. In particular, it will be shown later that, in presence of interfaces between ordinary dielectric regions where $\sqrt{\mu}$ is purely real and metamaterial regions with purely imaginary $\sqrt{\mu}$, the operator $\mathcal{O}$ remains nonHermitian [6].

The goal of the present paper is to demonstrate that the appearance of folded bands in metamaterial photonic crystals is a direct consequence of their essential non-Hermiticity. To this end, we will start by studying the simplest possible crystal structure, namely a onedimensional (1D) periodic stack of right- and left-handed materials. Several anomalous features of electromagnetic wave propagation have already been observed in this model $7-10$. Here we observe that the $1 \mathrm{D}$ model exhibits the so-called $\mathcal{P} \mathcal{T}$ symmetry [11] and, making use of this recently developed concept, we will explain the presence of folded bands in the spectrum of this model. Similar reasoning will be later applied to two-dimensional (2D) metamaterial crystals studied in [2, 3].

We assume that the 1D stack consists of materials $a$ and $b$ characterized by $\varepsilon_{i}, \mu_{i}$, refractive indices $n_{i}=$ $\sqrt{\varepsilon_{i} \mu_{i}}$, impedances $Z_{i}=\sqrt{\mu_{i} / \varepsilon_{i}}$, and thicknesses $\ell_{i}$, where $i=a, b$. All material parameters are assumed to be real and frequency-independent. The frequency spectrum of transverse electromagnetic waves, which propagate perpendicularly to the slabs with wave vector $q$, can be determined from the implicit equation 12

$$
f(\omega) \equiv \frac{1}{2}(A+1) \cos \omega \tau_{+}-\frac{1}{2}(A-1) \cos \omega \tau_{-}=\cos q \ell,
$$

where $\ell=\ell_{a}+\ell_{b}$ is the length of the unit cell, $\tau_{ \pm}=\tau_{a} \pm \tau_{b}$ with $\tau_{i}=n_{i} \ell_{i}$, and $A=\left(Z_{a} / Z_{b}+Z_{b} / Z_{a}\right) / 2>1$ is the impedance mismatch between the slabs $a$ and $b$.

In ordinary photonic crystals with $\varepsilon_{i}>0$ and $\mu_{i}>0$ we have $\tau_{+}>\left|\tau_{-}\right| \geq 0$. Therefore the larger-amplitude first term of $f(\omega)$ oscillates faster than the smaller-amplitude second term. Let us denote the positions of local extrema of the function $f(\omega)$ as $\omega^{*}$. One can check easily [13] that $\left|f\left(\omega^{*}\right)\right| \geq 1$ and from here it follows that no folded bands can be present in the spectrum. This was of course to be expected, since the wave equation of an ordinary photonic crystal can be Hermitized.

Now let us assume that the slab $a$ is an ordinary dielectric with $\varepsilon_{a}>0$ and $\mu_{a}>0$, whereas the slab $b$ is made from a metamaterial with $\varepsilon_{b}<0, \mu_{b}<0$, and $n_{b}<0$. In this case $\tau_{-}>\left|\tau_{+}\right| \geq 0$ and it is the smalleramplitude second term of $f(\omega)$ which oscillates faster than the larger-amplitude first term. As shown explicitly in Fig. 2(a), then the values of $f\left(\omega^{*}\right)$ may lie within the interval $(-1,1)$, and as a result folded bands can form in the spectrum. Such folded bands have been observed previously for oblique wave propagation 10, 14. Similarly as in the case of 2D metamaterial photonic crystals [3], also in the $1 \mathrm{D}$ case folded bands form only in a subset of the parameter space $\left(A, \beta=\tau_{-} / \tau_{+}\right)$of Eq. (3).

Since Eq. (3) does not shed light on the mathematical structure of the 1D problem, we shall restate its basic equations. The wave equation Eq. (1) reads piecewise

$$
-n_{i}^{-2} H^{\prime \prime}=\omega^{2} H
$$

If we take the center of the slab $a$ as the origin, then the boundary conditions at the interfaces $\xi= \pm \ell_{a} / 2$ between the slabs require

$$
H\left(\xi_{-}\right)=H\left(\xi_{+}\right), \quad E\left(\xi_{-}\right)=E\left(\xi_{+}\right),
$$

where $\xi_{-}$and $\xi_{+}$are infinitesimally shifted from $\xi$ to the left and right, respectively, and $E\left(\xi_{ \pm}\right)=H^{\prime}\left(\xi_{ \pm}\right) / \varepsilon\left(\xi_{ \pm}\right)$. Moreover, from the Bloch theorem follows an additional boundary condition

$$
H(\ell / 2)=e^{i q \ell} H(-\ell / 2) .
$$

The boundary-value problem which we have to solve is defined by Eqs. (4566).

Let us prove that the operator $\mathcal{O}$ for the $1 \mathrm{D}$ problem defined by Eqs. 456 is not Hermitian. To this end, let us define magnetic and "electric" fields corresponding to $h(x), H_{h}(x)=h(x) / \sqrt{\mu(x)}$ and $E_{h}(x)=$ $H_{h}^{\prime}(x) / \varepsilon(x)$. If $\mathcal{O}$ was Hermitian, then the quantity $D=\int_{-\ell / 2}^{\ell / 2} d x\left[g^{*}(x) \mathcal{O} h(x)-(\mathcal{O} g(x))^{*} h(x)\right]$ should be zero for any pair of functions $h(x)$ and $g(x)$, both of which generate fields $H_{h}, E_{h}$, and $H_{g}, E_{g}$ satisfying the boundary conditions Eqs. (56). Integrating per parts and taking proper care of the boundary terms, we find that $D=\Delta\left(-\ell_{a} / 2\right)+\Delta\left(\ell_{a} / 2\right)$, where

$$
\begin{aligned}
\Delta(\xi) & =H_{h}(\xi)\left[E_{g^{*}}\left(\xi_{-}\right)-E_{g^{*}}\left(\xi_{+}\right)\right] \\
& +E_{h}(\xi)\left[H_{g^{*}}\left(\xi_{+}\right)-H_{g^{*}}\left(\xi_{-}\right)\right]
\end{aligned}
$$

are the interface contributions. Note that in a righthanded medium $\left[H_{g}(x)\right]^{*}=H_{g^{*}}(x)$ and $\left[E_{g}(x)\right]^{*}=$ $E_{g^{*}}(x)$, whereas in a left-handed medium $\left[H_{g}(x)\right]^{*}=$ 

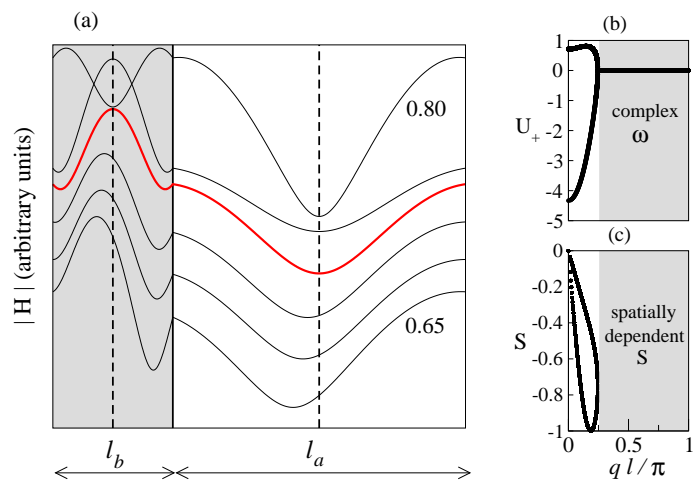

FIG. 3: (Color online) Observables for the 1D model with the same parameters as in Fig. 2. (a) Spatial distribution of $|H(x)|$. White and shaded regions correspond to layers $a$ and $b$, respectively. The vertical dashed lines denote the centers of the layers. Field distributions for the lowest folded band are shown for various values of $\cos q \ell$, from bottom to top: 0.650 , $0.700,0.710,0.719$ (critical value), 0.730 and 0.800 . Data are verticaly shifted for clarity. In $\mathcal{P} \mathcal{T}$-symmetric solutions the field $|H(x)|$ is even with respect to the center of any layer. $\mathcal{P} \mathcal{T}$ symmetry-breaking solutions lack this symmetry 18 . (b) The integral quantity $U_{+}$as a function of $q$. Note that $U_{+}=0$ for $\mathcal{P} \mathcal{T}$ symmetry-breaking solutions. (c) Real part of the Poynting vector, $S$, as a function of $q$. In all panels we use the normalization $\left|H\left(\ell_{a} / 2\right)\right|=1$.

$-H_{g^{*}}(x)$ and $\left[E_{g}(x)\right]^{*}=-E_{g^{*}}(x)$. It follows that in an ordinary photonic crystal the boundary conditions Eq. (5) imply that $E_{g^{*}}\left(\xi_{-}\right)=E_{g^{*}}\left(\xi_{+}\right)$and $H_{g^{*}}\left(\xi_{+}\right)=$ $H_{g^{*}}\left(\xi_{-}\right)$. Therefore $D=0$, as was to be expected. However, in a metamaterial photonic crystal $E_{g^{*}}\left(\xi_{-}\right)=$ $-E_{g^{*}}\left(\xi_{+}\right)$and $H_{g^{*}}\left(\xi_{+}\right)=-H_{g^{*}}\left(\xi_{-}\right)$. Thus $D \neq 0$ and hence the operator $\mathcal{O}$ is non-Hermitian.

Therefore the next question we should ask is: if the boundary value problem Eqs. 4 4 56 is non-Hermitian, how can its spectrum be real at all?

In order to answer this question, let us define operators of "parity" $\mathcal{P}$ and "time-reversal" $\mathcal{T}$ by $(\mathcal{P} H)(x)=$ $H(-x)$ and $(\mathcal{T} H)(x)=H^{*}(x)$, respectively. We find that Eqs. (45) are invariant under the action of both $\mathcal{P}$ and $\mathcal{T}$, while Eq. (6) is invariant only under the combined antilinear operator $\mathcal{P} \mathcal{T}$. Therefore the $1 \mathrm{D}$ model is $\mathcal{P} \mathcal{T}$-symmetric [15, as also observed for a somewhat similar boundary-value problem studied in [16].

Since $\mathcal{P}^{2}=1$ and $\mathcal{T}^{2}=1$, the eigenvalues of the antilinear operator $\mathcal{P} \mathcal{T}$ are $\lambda=e^{i \varphi}$, where $\varphi$ is real [11. Therefore a $\mathcal{P} \mathcal{T}$-symmetric solution should satisfy

$$
H^{*}(-x)=e^{i \varphi} H(x) .
$$

If the $\mathcal{P} \mathcal{T}$ symmetry is not broken, i.e. if the eigenstates of the wave equation are simultaneously also eigenstates of $\mathcal{P} \mathcal{T}$, the eigenvalues $\omega^{2}$ have to be real [11. On the other hand, if the eigenstates of the wave equation spontaneously break the $\mathcal{P} \mathcal{T}$ symmetry, then the eigenfrequencies corresponding to $H_{1}(x)$ and $H_{2}(x)=\mathcal{P} \mathcal{T} H(x)$

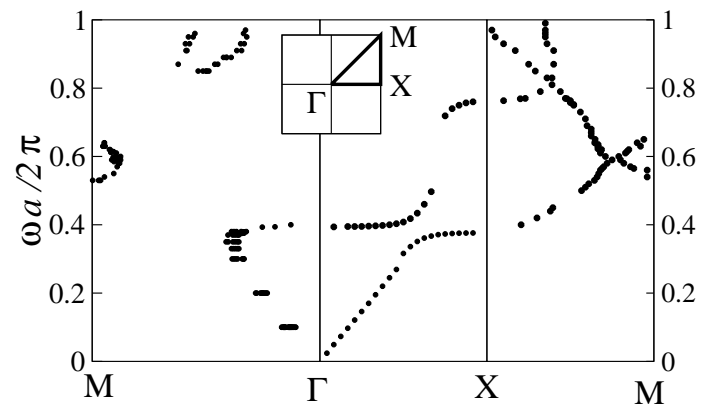

FIG. 4: Dispersion relations for the 2D model with cylinder radius $R=0.27 a$ along special lines in the $2 \mathrm{D}$ Brillouin zone (see inset). Electric field is polarized along the cylinders. Note that in the $\Gamma M$ direction, the two lowest bands are already folded. In the $X M$ and $\Gamma M$ directions, a third band enters the studied frequency range.

become complex conjugate. This is explicitly shown in Fig. 2(c), which has been obtained by solving Eq. (3) under the assumption of a complex frequency $\omega=\omega_{1}+i \omega_{2}$. Such pairs of eigenvalues have been observed previously in the $1 \mathrm{D}$ problem 9 and also in other $\mathcal{P} \mathcal{T}$-symmetric problems [17. Note that the complex-frequency solutions appear exactly for those values of momentum $q$, where the band folding has been observed. Therefore, if we allow for complex-valued frequencies, the number of eigenvalues does not vary with $q$.

We have checked numerically that for real-frequency solutions, the magnetic field does obey Eq. (7). On the other hand, according to Eq. (7), a $\mathcal{P} \mathcal{T}$-symmetric solution should satisfy $|H(-x)|=|H(x)|$. But Fig. 3(a) clearly shows that this criterion is not satisfied for complex-frequency solutions, thereby proving explicitly that they break the $\mathcal{P} \mathcal{T}$ symmetry.

Finally, we will turn back to the case of the square array of metamaterial cylinders with lattice constant $a$. Let us study electromagnetic waves with the electric field parallel with the cylinders and the $z$ axis. Then the wave equation reads piecewise $n_{i}^{-2} \triangle E=\omega^{2} E$ with a $2 \mathrm{D}$ Laplacian $\triangle$. The boundary conditions require the continuity of $E(x, y)$ and $E_{n}^{\prime}(x, y) / \mu(x, y)$ across the cylinder surfaces, where $E_{n}^{\prime}$ denotes a normal derivative. Moreover, a Bloch wave with wave vector $\vec{q}=\left(q_{x}, q_{y}\right)$ should satisfy the boundary conditions

$$
\begin{aligned}
& E(a / 2, y)=e^{i q_{x} a} E(-a / 2, y), \\
& E(x, a / 2)=e^{i q_{y} a} E(x,-a / 2) .
\end{aligned}
$$

Let us define the following $2 \mathrm{D}$ generalizations of the operators of "parity" $\mathcal{P}$ and "time-reversal" $\mathcal{T}$ : $(\mathcal{P} E)(x, y)=E(-x,-y)(\mathcal{T} E)(x, y)=E^{*}(x, y)$. Since the geometrical structure is $\mathcal{P}$-invariant and since the material parameters $\varepsilon_{i}, \mu_{i}$ and $n_{i}$ are taken to be real, the wave equation and the boundary conditions inside the unit cell are invariant under both, parity $\mathcal{P}$ and time- 

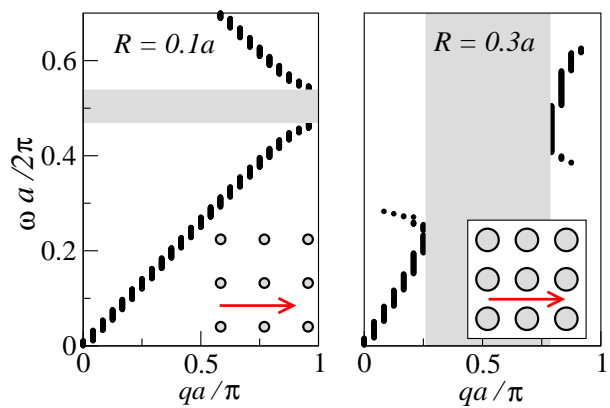

FIG. 5: (Color online) Dispersion relations along $\Gamma X$ for the 2D model with cylinder radii different from Fig. 1. The shaded regions highlight the frequency gap (with complex momentum solutions inside) in the left panel, and the momentum gap (with complex frequency solutions inside) in the right panel. Note the space - time duality of the two regions.

reversal $\mathcal{T}$. However, similarly as in the $1 \mathrm{D}$ example studied before, the Bloch boundary condition Eq. $(8)$ is preserved only under the action of the combined operator $\mathcal{P} \mathcal{T}$. Consequently, our $2 \mathrm{D}$ boundary value problem is again invariant under the action of the operator $\mathcal{P} \mathcal{T}$.

Once the non-Hermiticity and $\mathcal{P} \mathcal{T}$ symmetry of the $2 \mathrm{D}$ problem have been established, the reality of the spectrum in the $\mathcal{P} \mathcal{T}$-symmetric sector of eigenstates follows. In analogy with the 1D case, we conjecture that in those regions of the Brillouin zone where the folded bands disappear, there exist pairs of complex-frequency solutions in the spectrum. Unfortunately, since such states can not be seen in a numerical transmission experiment [3], we can not present a direct proof of their existence.

In the $2 \mathrm{D}$ problem, the critical value of the cylinder radius $R_{c}$, above which folded bands are formed, may depend on the direction in momentum space. In fact, Fig. 4 shows that for instance for $R=0.27 a$, the two lowest bands are already folded in the $\Gamma M$ direction.

It turns out that one can find a simple criterion for the appearance of folded bands. In fact, from the Maxwell equations in the frequency domain, $\operatorname{rot} \vec{E}=i \omega \vec{B}$ and $\operatorname{rot} \vec{H}^{*}=i \omega^{*} \vec{D}^{*}$, after multiplication by $\vec{H}^{*}$ and $\vec{E}$, respectively, follows the identity

$$
i \omega_{1} u_{-}+\omega_{2} u_{+}+\operatorname{div} \vec{S}=0,
$$

where $u_{ \pm}=\vec{E} \cdot \vec{D}^{*} \pm \vec{B} \cdot \vec{H}^{*}$ and $\vec{S}=\vec{E} \times \vec{H}^{*}$. Note that this identity holds also in dispersive and/or lossy media and it represents a generalization of Poynting's theorem for harmonic fields [19]. In our case of lossless media the quantities $u_{ \pm}$are both real. Let us integrate Eq. (9) over the area of an elementary cell of the photonic crystal. The Bloch boundary conditions Eq. (8) imply that $\int_{\text {cell }} d^{2} x \operatorname{div} \vec{S}=0$. Therefore $i \omega_{1} U_{-}+\omega_{2} U_{+}=0$ must hold, where $U_{ \pm}=\int_{\text {cell }} d^{2} x\left[\varepsilon|\vec{E}|^{2} \pm \mu|\vec{H}|^{2}\right]$. Considering the real part of this condition for $\mathcal{P} \mathcal{T}$ symmetry-breaking solutions with $\omega_{2} \neq 0$, we find that $U_{+}=0$ must hold, as confirmed for the 1D problem in Fig. 3(b). Similar criteria for the occurence of folded bands have been found in 2 by different reasoning. Note that at the verge of $\mathcal{P} \mathcal{T}$ symmetry breaking, the group velocity diverges and $U_{+}$ vanishes. Explicit calculation for the 1D problem shows that the real part of $\vec{S}$ stays finite here, see Fig. 3(c).

Before concluding let us observe that folded bands are closely related to the frequency gap in periodic systems. In fact, it is well known that within the frequency gap, there exist only solutions with a complex wave-vector. On the other hand, folded bands imply the existence of a momentum gap, inside which there appear only states with a complex frequency. Thus the momentum gap is a space - time dual of the frequency gap, see Fig. 5 .

In conclusion, we have explained the physical origin of folded bands, or momentum gaps, in spectra of metamaterial crystals. Two ingredients are necessary for their appearance: first, the boundary-value problem has to be essentially non-Hermitian, and second, the possible reality of its spectrum has to be guaranteed by additional symmetry, such as the $\mathcal{P} \mathcal{T}$ symmetry in the metamaterial case. In systems fulfilling these assumptions, momentum gaps should be commonplace.

We thank V. Balek and M. Mojžiš for helpful discussions. This work was supported by the Slovak Research and Development Agency under the contract No. APVV0108-11 and by the Agency VEGA under the contracts No. 1/0372/13 and No. 1/0904/15.

[1] K. Sakoda, Optical Properties of Photonic Crystals, Berlin, Heidelberg: Springer (2005).

[2] P. Y. Chen et al, New J. Phys. 13053007 (2011).

[3] P. Markoš, unpublished. The method has been described in P. Markoš, preprint arXiv:1501.05125.

[4] It is worth pointing out that folded bands have been observed also earlier, see, e.g., D. Toader and S. John, Phys. Rev. E 70046605 (2004) and D. Hermann et al, Phys. Rev. B 77035112 (2008). However, in those papers systems with frequency-dependent material parameters were studied. Therefore, from the mathematical point of view, those authors did not study eigenvalue problems, and the presence of folded bands in spectra did not lead to the sort of questions which we address.

[5] J. D. Joannopoulos et al., Photonic Crystals: Molding the Flow of Light, 2nd ed. Princeton: Princeton University Press (2008).

[6] Since we are dealing with a metamaterial crystal, Hermitization of the wave equation for $\vec{E}$ in terms of the substitution $\vec{E}=\vec{e} / \sqrt{\varepsilon}$ is plagued by the same problem.

[7] I. S. Nefedov and S. A. Tretyakov, Phys. Rev. E 66, 036611 (2002).

[8] Jensen Li et al., Phys. Rev. Lett. 90, 083901 (2003).

[9] Liang Wu, Sailing He, and Linfang Shen, Phys. Rev. B 67, 235103 (2003)

[10] D. Bria et al., Phys. Rev. E 69, 066613 (2004). 
[11] For a review, see C. M. Bender, Contemporary Physics 46, 277 (2005).

[12] P. Yeh, Optical Waves in Layered Materials, Hoboken, New Jersey: Wiley (2005).

[13] To this end it suffices to note that for frequencies $\omega_{n}=$ $n \pi / \tau_{+}$, where $n$ is an integer, we have $\left|f\left(\omega_{n}\right)\right| \geq 1$. Moreover, $f\left(\omega_{n}\right)$ exhibits even-odd oscillations with $n$. But since the second term in $f(\omega)$ oscillates with a longer period, in between $\omega_{n}$ and $\omega_{n+1}$ there will be at most one extreme of $f(\omega)$, and therefore $\left|f\left(\omega^{*}\right)\right| \geq\left|f\left(\omega_{n}\right)\right| \geq 1$.

[14] Also the much debated zero- $\bar{n}$ gaps [8] are associated with a special case of folded bands. In fact, the zero- $\bar{n}$ condition is equivalent to $\tau_{+}=0$ and in this case Eq. (3) has solutions only for $q=0$ and $\omega \tau_{-}=2 m \pi$, where $m$ is an integer.

[15] In previous work, the concept of $\mathcal{P} \mathcal{T}$ symmetry has been exploited in optics from a quite different perspective, making use of the formal analogy between quantum mechanics and the paraxial approximation. The $\mathcal{P} \mathcal{T}$ symmetry was achieved by judicious spatial modulation of loss and gain, see e.g. C. E. Rüter et al., Nat. Phys. 6, 192 (2010). The closest in spirit to ours is the paper L. Ge and H. E. Türeci, Phys. Rev. A 88, 053810 (2013), which also studies metamaterial crystals (with different symmetry) using the concept of $\mathcal{P} \mathcal{T}$ symmetry. That paper is limited only to $1 \mathrm{D}$ systems, however, and, more importantly, it does not study the eigenvalue problem.

[16] D. Krejčiřík, H. Bíla, and M. Znojil, J. Phys. A: Math. Gen. 39, 10143 (2006).

[17] For a recent example, see A. Mostafazadeh, J. Phys. A: Math. Theor. 44, 375302 (2011).

[18] In the $\mathcal{P} \mathcal{T}$ symmetry-breaking sector, we plot $|H(x)|$ for only one solution for every value of $q$. The minima of $|H(x)|$ shift to the left from the center of layer $a$. The other solution (not shown), which corresponds to a complex-conjugate frequency, exhibits an opposite shift.

[19] J. D. Jackson, Classical Electrodynamics, $3^{\text {rd }}$ ed., New York: John Wiley (1999). 